\title{
Magnetic Full Adder Based on NDR-enhanced Anomalous Hall Effect
}

\author{
Ziyao $\mathrm{Lu}^{1}$, Hongming Mou ${ }^{1}$, Yuchen $\mathrm{Pu}^{1}$, Yan Wen ${ }^{2}$, Xixiang Zhang ${ }^{2}$ and Xiaozhong Zhang ${ }^{1^{*}}$ \\ 1. School of Materials Science and Engieering and Key Laboratory of Advanced Materials (MOE), Tsinghua University, Beijing \\ 100084, China. \\ 2. Division of Physical Science and Engineering, King Abdullah University of Science and Technology (KAUST), Thuwal 239955, \\ Kingdom of Saudi Arabia
}

\begin{abstract}
Received 1 Apr 2020, revised 15 Apr 2020, accepted 20 Apr 2020, published 1 Jun 2020, current version 15 Jun 2020. (Dates will be inserted by IEEE; "published" is the date the accepted preprint is posted on IEEE Xplore ${ }^{\circledR}$; "current version" is the date the typeset version is posted on Xplore ${ }^{\circledR}$ ).

Abstract-Spintronic logic devices have attracted attention because of the prospect of breaking the von-Neumann bottleneck through nonvolatile in-memory computing. Although varieties of spin Boolean logic gates have been proposed, spintronic arithmetic logic units such as adders have not been extensively studied because of the difficulties in application of the cascade method of CMOS-based logic in spintronic devices. Here, we experimentally demonstrated a spintronic full adder based on anomalous Hall effect and geometrical tuning magnetization switching driven by spin-orbit torque. The anomalous Hall effect of magnetic bits was enhanced by nonlinear elements with $\mathrm{N}$ type negative differential resistance to control the ON/OFF state of MOSFETs which determined the write voltage of the memory unit. The magnetization of the memory bits in the memory unit were switched one by one as write voltage increased because of geometry difference. The order of magnetization switching caused the response of the anomalous Hall voltage of the memory unit to the input configurations to conform with the logic function of full adder. Computation function of full adder combined with memory writing was experimentally realized with only 7 magnetic bits and 2 steps. The reduced number of magnetic bits and time steps indicated efficiency of space and time of our device which beneficial for practical applications.
\end{abstract}

Index Terms-Spin Electronics, Electrical control of spin, Magnetic logic devices

\section{$1 \quad$ I. INTRODUCTION}

2 Conventional CMOS-based computer with processor built on 3 volatile semiconductor logic elements requires frequent data 4 transfer between CPU and external memory [Luo 2017]. As the 5 line width of semiconductor devices shrinks, the drawback of such 6 von-Neumann architecture in performance and power dissipation 7 has become nonnegligible [Joshi 2020]. On one hand, the time 8 delay on the BUS and the performance gap between processor and 9 nonvolatile memory have limited the operation speed. On the 10 other hand, the energy consumption in the process of data 11 communication leads to extra power dissipation [Backus 1983, 12 Liu 2005]. Spintronics is one of the emerging fields that have the 13 potential to break through the von-Neumann bottleneck by 14 combining data processing and storage within a single integrated 15 platform [Joshi 2020, Dieny 2020]. Such prospect has motivated 16 research on nonvolatile spintronic logic devices compatible with 17 modern semiconductor technologies such as electric-field 18 controlled spin logic [Li 2018, Baek 2018], magnetic logic gates 19 based on magnetic tunnel junctions (MTJ) [Zabihi 2019], 20 magnetic domain-wall (DW) logic [Allwood 2005, Omari 2019, 21 Zheng 2020, Luo 2020] and reconfigurable magnetic gates based 22 on anomalous Hall effect (AHE) enhanced by nonlinear elements 23 [Luo 2017, Lu 2021, Pu 2020, Pu 2021]. Although varieties of 24 spintronic Boolean logic gates have been proposed, problems still

Corresponding author: Xiaozhong Zhang

Digital Object Identifier: 10.1109/LMAG.XXXX.XXXXXXX (inserted by IEEE).
25 remain in experimental demonstration of more complex 26 computing elements such as full adder. For electric-field 27 controlled spin logic, despite its low power consumption 28 benefitting from the field-controlled mechanism, the current logic 29 implementation scheme is incapable of practical computing tasks 30 because of the poor cascading property [Li 2018, Baek 2018]. In 31 MTJ-based magnetic logic circuits, adders and other arithmetic 32 logic elements were constructed by cascading of NAND gates 33 through sequential read-out and data writing of MTJs [Zabihi 34 2019]. Because each step of cascading would cost one clock cycle 35 of operation time, the time efficiency of complex combinational 36 logic functions requiring several steps of cascading was limited. 37 DW-based combinational logic circuits perform logic functions by 38 connecting majority gates based on propagation of DWs with 39 networks of ferromagnetic nanowires [Luo 2020]. Although such 40 method could cascade logic gates directly, it is not suitable for 41 logic circuits with large scale because the operation time is limited 42 by the speed of DW motion. AHE-based spin logic enhances the 43 AHE of magnetic multilayers with perpendicular magnetic 44 anisotropy (PMA) by nonlinear elements with negative 45 differential resistance (NDR) [Luo 2017, Lu 2021, Pu 2020, Pu 46 2021]. Magnetic majority gates was realized by connecting three 47 PMA in parallel, and the enhanced AHE signal was transformed 48 into the write current of storage bits, which could serve as the 49 input of logic gates on the next level. Thus, complex 50 combinational logic functions such as adders were performed by 51 cascading of majority gates. However, in such method of logic 52 implementation where logic functions relied on interconnection of 53 input bits, one input bit could only be used in one logic gate at the 
1 same time. The poor fan-out property increased the time delay of 2 complex logic operations. Moreover, the number of bits required 3 to complete the logic operation far exceeded the number of 4 variables in the logic operation itself, resulting in redundancy of 5 space occupation in memory for the logic operations. In order to 6 improve the fan-out property and reduce the number of magnetic 7 bits involved in logic operations, we propose here a new 8 implementation of AHE-based spin logic. A full adder combined 9 with reconfigurable Boolean logic gate covering logic operations 10 AND, OR, NAND and NOR was demonstrated experimentally. 11 Contrary to previous full adders, the inputs of our device are 12 insulated from each other and each input could be shared by 13 multiple logic gates. The number of magnetic bits needed was 14 reduced by $50 \%$ and the required clock cycle was reduced by $33 \%$ 15 compared with the previous AHE-based spintronic full adder $[\mathrm{Pu}$ 16 2021].

17

18

\section{EXPERIMENT}

19 The three-terminal magnetic bits in our device were fabricated 20 from magnetic multilayer Ta $(3 \mathrm{~nm}) / \mathrm{Co}_{40} \mathrm{Fe}_{40} \mathrm{~B}_{20}(0.7 \mathrm{~nm}) / \mathrm{MgO}$ $21(1 \mathrm{~nm})$ with $\mathrm{SiO}_{2}$ capping layer of $5 \mathrm{~nm}$ deposited on $\mathrm{Si}$ substrate 22 with $300 \mathrm{~nm}$ of thermally oxidized $\mathrm{SiO}_{2}$. After sputtering (Rotaris 23 Singulus $)$, the multilayer was annealed at $220{ }^{\circ} \mathrm{C}$ in vacuum $(<2$ $\left.24 \times 10^{-4} \mathrm{~Pa}\right)$ for 1 hour to achieve perpendicular magnetic 25 anisotropy. The magnetic bits were then patterned by UV 26 lithography and Ar-ion milling. Ti $(10 \mathrm{~nm}) / \mathrm{Au}(50 \mathrm{~nm})$ was used 27 for electric contacts. Apart from the magnetic elements, other 28 components of our device including the NDR elements and 29 MOSFETs were available on the market. Measurements to 30 demonstrate the logic and memory functions of the device, 31 including electrical manipulation of magnetization direction of the $32 \mathrm{CoFeB}$ layer and measuring the response of output voltage to 33 different magnetization states of input bits, were conducted using 34 Keithley 2400 and Keithley 2182A at room temperature, with the 35 external magnetic field provided by an electromagnet.

36

37

\section{RESULT AND DISCUSSION}

38 The AHE-based spintronic full adder was mainly composed of 39 two parts: logic inputs and memory unit consisted of a group of 40 parallel-connected magnetic bits with different length (Fig. 1). 41 The two parts were connected by three n-channel enhancement 42 MOSFETs and resistors.

43 The logic inputs comprised three magnetic bits and three pairs 44 of $\mathrm{N}$ type NDR elements (Fig. 1). The three-terminal magnetic 45 bits were fabricated from ferromagnetic multilayers $46 \mathrm{Ta} / \mathrm{CoFeB} / \mathrm{MgO}$ with perpendicular magnetic anisotropy. Due to 47 the AHE, the voltage at the top electrode was dependent on the 48 magnetization of the magnetic bit. However, the magnitude of the 49 difference of voltage was too small for logic functions because of 50 the risk of logic errors. In our device, pairs of $\mathrm{N}$ type NDR 51 semiconductor elements were used to solve this problem. By 52 connecting NDR pairs to the magnetic bits (Fig. 1), positive 53 feedback was introduced to the circuit. When the magnetization of 54 the magnetic bits was upward (" 0 " state), the voltage between the
55 top and right electrode $V_{O}$ exceeded the voltage between the left 56 and top electrode $V_{i n}-V_{O}$. As a result, the right NDR would reach 57 the negative slope region earlier than the left NDR. In this region, 58 increase of the voltage on the NDR caused the current to decrease, 59 leading to increase of resistance. The increase of resistance would 60 lead to further increase of voltage, such positive feedback resulted 61 in a large output voltage $V_{O H}$. On the contrary, in the case where 62 the magnetization pointed downward ("1" state), $V_{O}$ was smaller 63 than $V_{i n}-V_{O}$ because of AHE. The left NDR would first reach 64 negative slope region and the positive feedback resulted in a large $65 V_{i n}-V_{O}$. Since the logic input circuit was driven by voltage source $66 V_{i n}$, a large $V_{i n}-V_{O}$ indicated a low voltage output $V_{O L}$. As a result, 67 the NDR pair enhanced the difference between $V_{O}$ and $V_{i n}-V_{O}$ 68 caused by AHE, which ensured the output ratio to be large enough 69 for logic applications (Fig. 2(a)). Detailed analysis about the 70 mechanism of N-NDR enhancement of anomalous Hall voltage 71 was performed by load-line analysis [ $\mathrm{Pu} 2021]$. The three-terminal 72 magnetic bit could be modelled by $\Delta$-type resistor network 73 comprised of three resistors $R_{\text {Left }}^{E f f}, R_{\text {Right }}^{E f f}$ and $R_{\text {Mid }}^{E f f}$. According to

74 Kirchhoff's Law, the voltage and current in $R_{\text {Left }}^{\text {Eff }}, R_{\text {Right }}^{\text {Eff }}$ and the 75 NDR pair followed the equations below:

$\begin{array}{lcc}76 & I_{0}=I_{\text {in }}-V_{\text {in }} / R_{\text {Mid }}^{E f f} & \text { (Eq. 1) } \\ 77 & V_{\text {in }}=V_{\text {Left }}+V_{\text {Right }} & \text { (Eq. 2) } \\ 78 & I_{0}=I_{\text {Left }}^{N D R}+V_{\text {Left }} / R_{\text {Left }}^{E f f}=I_{\text {Right }}^{N D R}+V_{\text {Right }} / R_{\text {Right }}^{E f f} & \text { (Eq. 3) }\end{array}$

79 Io was the current in the upper branch of the circuit $\left(R_{\text {Leff }}^{E f f}\right.$, $80 R_{\text {Right }}^{E f f}$ and the NDR pair). The resistance of the resistors was 81 dependent on the magnetization of the magnetic bits because of 82 the AHE. When the magnetization was upward (downward), $R_{R i g h t}^{E f f}$ 83 was larger (smaller) than $R_{\text {Left }}^{\text {Eff }}$. Besides the equations above, the 84 current and voltage in the NDRs should also follow the I-V 85 characteristics of NDR.

$86 \quad I_{\text {Left }(\text { Right })}^{N D R}=I^{N D R}\left(V_{\text {Left }(\text { Right })}\right) \quad$ (Eq. 4)

87 The current and voltage of NDRs was presented by the 88 solutions of Eq. 3 and Eq. 4 which could be derived by load line 89 analysis. The $\mathrm{N}$-shaped $I-V$ curve represented the $I-V$ 90 characteristics of NDRs and the straight lines were drawn 91 according to Eq. 3. The straight lines and the intersection points in 92 the figures were referred to as load lines and working points 93 respectively. The different slopes of the load lines were caused by 94 the magnetization-dependent resistance of $R_{\text {Left }}^{E f f}$ and $R_{\text {Right }}^{E f f}$. The 95 analysis below was based on the case of magnetization upward 96 and the opposite case could be analyzed with similar method.

97 When $V_{\text {in }}$ started to increase from zero, both working points 98 were in the positive differential resistance region of the $\mathrm{N}$-shaped 99 curve and moved upward and rightward (Fig. 3(b)). Once the 100 upper load line reached the maximum point of the curve, the two 101 working points would move in different directions as $V_{\text {in }}$ 102 continued to increase (Fig. 3(c)). At this stage, as the $x$ 103 coordinates of the working points represented the voltage of the 104 NDRs, the separation of working points caused the difference 105 between $V_{\text {Left }}$ and $V_{\text {Right }}$ to increase rapidly, which could explain 106 the bifurcation $V_{O}$ in Fig. 2(a). Such bifurcation enlarged the 107 magnetic response of $V_{O}$. As a result, the magnetization of input 
1 bits was transformed to the magnitude of $V_{O}$ with large output 2 ratio so that $V_{O}$ could control the writing of the memory unit in 3 Fig. 1.

4 As mentioned above, when the magnetization of the input bit 5 was upward (" 0 " state), the output voltage $V_{O}$ was in high voltage 6 state $V_{O H}$. On the contrary, when the magnetization of the input 7 bit was downward ("1" state), the output voltage $V_{O}$ was in low 8 voltage state $V O L$. The output voltage of input circuit served as the 9 gate voltage $V_{G S}$ of the MOSFETs with threshold gate voltage $10 V_{G S(T H)}$ satisfying $V_{O H}>V_{G S(T H)}>V_{O L}$. Thus, the ON/OFF state of 11 MOSFETs was determined by the magnetization of the magnetic 12 bits. Since the three input bits a, b and c respectively controlled 13 the ON/OFF states of the three MOSFETs, the write voltage $V_{\text {Write }}$ 14 on the memory unit with three parallel-connected memory bits 15 was determined by the magnetization of input bits (Fig. 2(b)). The 16 experiment result showed that the value of $V_{\text {Write }}$ was related to the 17 number of "0"s and "1"s in the three input terminals. When all 18 three inputs were "1", the output voltage $V_{O}$ of all the inputs were 19 low and the MOSFETs were in the OFF state. Since the $R_{D S}$ of 20 OFF-state MOSFETs was much larger than the resistance of the 21 memory unit, the voltage $V_{\text {Write }}$ applied on the memory unit was 22 very low. When one of the inputs was " 0 ", the output voltage $V_{O}$ 23 was in the high voltage state $V O H$ and the MOSFET connected to 24 this input was switched ON. In this case, the write voltage $V_{\text {Write }}$ 25 depended on the resistor R connected to the MOSFET. Since the 26 resistance of $\mathrm{R}$ was much smaller than $R_{D S}$ of OFF-state MOSFET, $27 V_{\text {Write }}$ increased. As the number of " 0 "s of the inputs increased 28 from 0 to 3 , the more resistors were connected in the write circuit. 29 Since $V_{D D}$ was constant and the resistors could be considered as 30 parallel-connected, the voltage $V_{\text {Write }}$ applied on the memory unit 31 increased stepwise since the resistors were connected in parallel 32 (Fig. 2(b)). Such dependency of $V_{\text {Write }}$ on the inputs set up the 33 relation between the number of " 0 "s of the inputs and the write 34 voltage. Thus, the logic function of the device depended on the 35 state of memory unit under different $V_{\text {Write }}$.

36 The memory unit consisted of three parallel-connected 37 magnetic bits sharing the same width (W) but had different length 38 (L). According to previous work of spin - orbit - torque (SOT) 39 driven magnetization switching [Liu 2012], the charge current in $40 \mathrm{Ta}$ layer of the magnetic bits generated out-of-plane spin current 41 which would switch the magnetization of the ferromagnetic $42 \mathrm{CoFeB}$ layer with the assistance of a constant external magnetic 43 field colinear with the current. The critical switching current of 44 magnetization reversal driven by spin-orbit torque was related to 45 the current density $J_{C}$, which was proportional to the electric field $46 V_{\text {write }} / L$. Thus, the difference in length among the magnetic bits 47 would lead to different critical switching voltage (Fig. 4). The 48 opposite chirality of magnetization reversal of the magnetic bit in 49 the middle was because the magnetic bit in the middle was 50 connected inversely to the other two bits. By comparing the 51 stepwise write voltage $V_{\text {Write }}$ (Fig. 2b) and the critical switching 52 voltage $V_{C}$ of the memory bits (Fig. 4), it could be found that with 53 the number of " 0 "s of the inputs increased from 0 to 3 , the 54 magnetization of the three bits would be reversed in sequence by 55 SOT (Fig. 5(a)). As a result, as the number of "0"s of the three 56 inputs increased, the memory unit experienced four different 57 magnetization states $(0,1,0),(1,1,0),(1,0,0)$, and $(1,0,1)$,
58 corresponding to number of " 0 "s of the inputs $0,1,2$ and 3 59 respectively. When focusing on the relation between the 60 magnetization state of the middle bit and the inputs, it could be 61 found that such relation matched with 3-input majority gate, 62 which served as CARRY output (Cout) of full adder.

63 In addition to the COut, the "SUM" (S) output also needed to be 64 given for a full adder. As Fig. 5(a) demonstrated, when the 65 number of " 0 "s in the three input bits a, b, and c increased from 0 66 to 3 , the magnetization of the memory unit changed in the order of $67(0,1,0),(1,1,0),(1,0,0)$, and $(1,0,1)$. The change of magnetization 68 state resulted in different anomalous Hall voltage when the AHE 69 of the three magnetic bits were superimposed by connecting them 70 in parallel with the same current direction (Fig. 5(b)). If the 71 positive and negative value of the Hall signal were regarded as 72 logic output "1" and "0" respectively, as the number of " 0 "s in the 73 three input bits $\mathrm{a}, \mathrm{b}$, and $\mathrm{c}$ increased from 0 to 3 , the output 74 changed in the sequence of ("0", "1", "0", “1"), which was 75 consistent with the truth table of the "SUM" output of a full adder. 76 Therefore, once the magnetization states of the memory unit was 77 determined by the three input bits $a, b$, and $c$, the computation 78 results of full adder including the "SUM" and "CARRY OUT" 79 were already stored in the memory unit in the form of the 80 magnetization of the middle bit and the whole memory unit 81 respectively, which indicated the completion of the computing 82 process of full adder. Moreover, the "SUM" output represented by 83 the overall anomalous Hall signal of the three magnetic bits of 84 memory unit could be rewritten into one magnetic bit, which 85 could serve as an input bit in subsequent logic operations when 86 cascade was needed. In the rewriting process, N-NDR pairs were 87 applied to enhance the output "SUM" signal (Fig. 6(a)), and the 88 enhanced output voltage $V_{\text {Out }}$ was also capable of controlling the $89 \mathrm{ON} / \mathrm{OFF}$ state of a MOSFET. Hence, the write voltage $V_{S}$ across 90 storage bit d (Fig. 6(b)(c)) was be determined by the 91 magnetization state of the memory unit and the "SUM" output 92 contained in the memory unit was written into the storage bit $\mathrm{d}$ 93 (Table 1), which was ready to serve as inputs of subsequent 94 magnetic logic operations.

95 Compared with the previous full adder [Pu 2021], the full adder 96 presented above has advantages in the following three aspects. 97 Firstly, in our AHE-based spintronic full adder, the memory unit 98 played a major role in the realization of the logic functions, 99 whereas in other AHE-based devices logic functions relied on the 100 interconnection between the input bits. Such design allowed the 101 function of memory unit to be no longer limited to storage of logic 102 outputs but also in logic applications. Since the memory unit 103 shared the task of logic, it was not necessary for our logic devices 104 to use a large number of input bits to implement complex logic 105 functions. Compared with previous AHE-based full adder [Pu 106 2021], the number of magnetic bits was reduced from 14 to 7 , 107 which was beneficial for decreasing the occupation of space. 108 Secondly, because of complicated cascade process, the previous 109 AHE-based full adder took three steps to accomplish an adding 110 computation. Because the inputs needed to be called repetitively 111 in the computation process, the data stored in the input bits could 112 not be changed throughout the process. As a result, the inputs 113 could only be redefined in an extra step after the computation 114 process, which was disadvantageous for time efficiency. In 
1 comparison, the computation process of the proposed full adder 2 consisted of two steps. In the first step, the magnetization of the 3 memory unit was reversed according to the inputs, storing the 4 output of the full adder in the memory unit. In the second step, the 5 computation result was transferred into a single magnetic bit in 6 convenience of cascade. During the second step the inputs could 7 be rewritten or redirected to prepare for the next computation task 8 assigned to the logic device allowing for pipe-line computations. 9 The reduction of computation steps and availability of pipi-line 10 computation indicated the advantage of our device in time 11 efficiency. Thirdly, our device conformed well the requirements 12 of parallel computation. As mentioned above, the logic functions 13 of our device were performed mostly in the process of writing 14 process where the logic input bits contributed equally and 15 independently. Since the logic function no longer relied on the 16 interconnection between input bits, the inputs acquired the ability 17 of fan-out, which allowed each of them be shared by multiple 18 logic units simultaneously. The fan-out ability of logic inputs was 19 important for parallel computations.

20 In conclusion, by introducing geometrical design into the 21 memory unit, we demonstrated a new nonvolatile spintronic full 22 adder based on NDR-enhancement of AHE. The design of 23 memory unit consisted of three magnetic bits different in length 24 allowed the logic functions to be performed in the writing process 25 instead of by complex interconnections of input bits. The 26 redistribution of logic and memory function led to optimization of 27 AHE-based full adder device in terms of space occupation, time 28 efficiency and ability of parallel computing, indicating 29 improvements of AHE-based spintronic logic devices aiming at 30 practical applications.

31

32

\section{ACKNOWLEGDMENT}

33 This work was sponsored by National Key R\&D Program of 34 China (Grant No.: 2017YFA0206202) and National Science 35 Foundation of China (Grant No.: 11674190). The authors thank 36 Beijing National Center for Electron Microscopy for providing 37 research facilities.

38

39

\section{REFERENCES}

40 Luo Z C, Lu Z Y, Xiong C Y, Zhu T, Wu W, Zhang Q, Wu H Q, Zhang X X, Zhang 41 X Z (2017), "Reconfigurable Magnetic Logic Combined with Nonvolatile 42 Memory Writing," Adv. Mat., vol. 29, pp. 6, doi: 10.1002/adma.201605027.

43 Joshi V K, Barla P, Bhat S, Kaushik B K (2020), "From MTJ Device to Hybrid 44 CMOS/MTJ Circuits: A Review," IEEE Access, vol. 8, pp. 194105-194146, 45 doi: $10.1109 / \mathrm{AC}$

46 Backus J (1983), "Can programming be liberated from the von Neumann style? A

47 functional style and its algebra of programs," in Programming languages: A 48 grand tour, Rockville, MD, USA: Computer Science Press, pp. 146-174.

49 Liu C C, Ganusov I, Burtscher M, Tiwari S (2005), "Bridging the processor-memory 50 performance gap with 3D IC technology," IEEE Design \& Test of Computers, 51 vol. 22, pp. 556-564, doi: 10.1109/mdt.2005.134.

52 Dieny B, Prejbeanu I L, Garello K, Gambardella P, Freitas P, Lehndorff R, Raberg W, 53 Ebels U, Demokritov S O, Akerman J J N E (2020), "Opportunities and 54 challenges for spintronics in the microelectronics industry," Nat. Elecron. vol. 55 3, pp. 446-459, doi. 10.1038/s41928-020-0461-5

56 Li X, Lee A, Razavi S A, Wu H, Wang K L (2018), "Voltage-controlled 57 magnetoelectric memory and logic devices," MRS Bulletin, vol. 43, pp. 97058977 , doi. $10.1557 / \mathrm{mrs} .2018 .298$
59 Baek S-h C, Park K-W, Kil D-S, Jang Y, Park J, Lee K-J, Park B-G (2018), 60 "Complementary logic operation based on electric-field controlled spin-orbit 61 torques," Nature Electronics, vol. 1, pp. 398-403, doi: 10.1038/s41928-01862 0099-8.

63 Zabihi M, Chowdhury Z I, Zhao Z, Karpuzcu U R, Wang J, Sapatnekar S S (2019), 64 "In-Memory Processing on the Spintronic CRAM: From Hardware Design to 65 Application Mapping," IEEE Transactions on Computers, vol. 68, pp. 115966 1173, doi: 10.1109/TC.2018.2858251.

67 Allwood D A, Xiong G, Faulkner C, Atkinson D, Petit D, Cowburn R (2005),

68 "Magnetic domain-wall logic," Science, vol. 309, pp. 1688-1692, doi: $6910.1126 /$ science. 1108813.

70 Omari K A, Broomhall T J, Dawidek R W, Allwood D A, Bradley R C, Wood J M,

71 Fry P W, Rosamond M C, Linfield E H, Im M Y (2019), "Toward 72 Chirality-Encoded Domain Wall Logic," Advanced Functional Materials, vol. 73 29, pp. 1807282, doi: 10.1002/adfm.201807282.

74 Zheng X, Wang J, Li G, Lu X, Li W, Wang Y, Chen L, Yin H, Wu J, Xu Y (2020),

75 "Paradigm of Magnetic Domain Wall-Based In-Memory Computing," ACS

76 Applied Electronic Materials, vol. 2, pp. 2375-2382, doi: 77 10.1021/acsaelm.0c00318.

78 Luo Z, Hrabec A, Dao T P, Sala G, Finizio S, Feng J, Mayr S, Raabe J, Gambardella 79 P, Heyderman L J (2020), "Current-driven magnetic domain-wall logic," Nature, 80 vol. 579, pp. 214-218, doi: 10.1038/s41586-020-2061-y

$81 \mathrm{Lu}$ Z, Xiong C, Mou H, Luo Z, Fang C, Wan C, Wu H, Zhang X, Zhang X (2021),

82 "Nonvolatile magnetic half adder combined with memory writing," Applied 83 Physics Letters, vol. 118, pp. 182402, doi: 10.1063/5.0048448.

$84 \mathrm{Pu}$ Y, Mou H, Lu Z, Nawaz S, Wang G, Zhang Z, Yang Y, Zhang X, Zhang X (2020), 85 "Speed enhancement of magnetic logic-memory device by insulator-to-metal

86 transition," Applied Physics Letters, vol. 117, pp. 022407, doi: $8710.1063 / 5.0013301$

$88 \mathrm{Pu} \mathrm{Y,} \mathrm{Lu} \mathrm{Z,} \mathrm{Mou} \mathrm{H,} \mathrm{Zhang} \mathrm{X,} \mathrm{Zhang} \mathrm{X} \mathrm{(2021),} \mathrm{"Ultrafast} \mathrm{and} \mathrm{ultralow} \mathrm{power}$ 89 voltage-dominated magnetic logic," submitted for publication.

90 Liu L, Pai C-F, Li Y, Tseng H W, Ralph D C, Buhrman R A (2012), "Spin-Torque 91 Switching with the Giant Spin Hall Effect of Tantalum," Science, vol. 336, pp. 92 555-558, doi: $10.1126 /$ science. 1218197

93

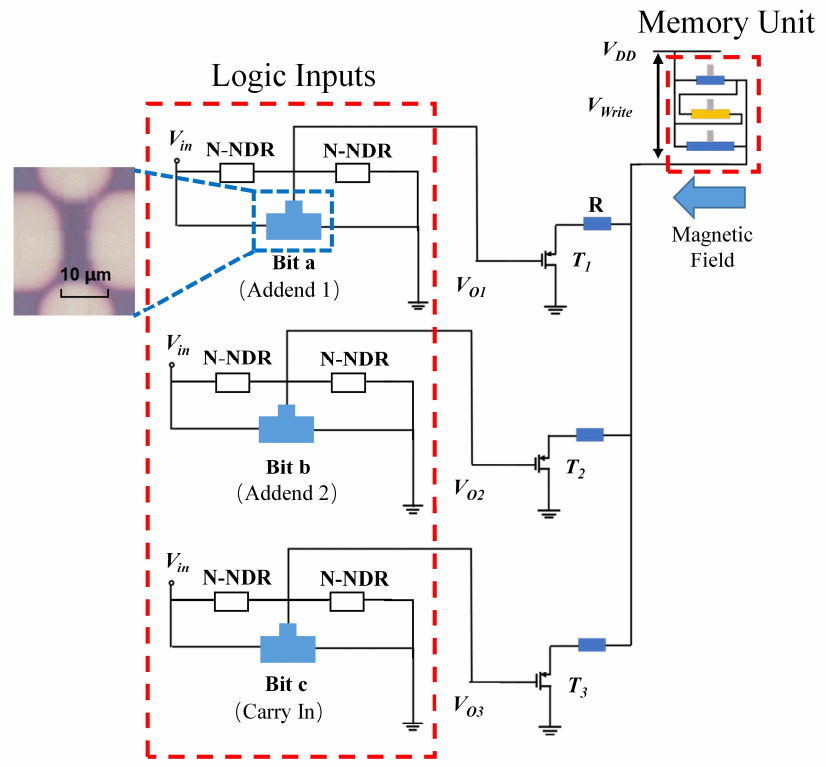

96 Fig. 1 Schematics of the spintronic full adder based on NDR97 enhanced AHE. Image of the magnetic bits in optical microscope 98 was presented. The bottom electrode of the magnetic bit was only 99 used to measure the current-induced reversal of magnetization and 100 was not involved in the logic operations.

101 
1
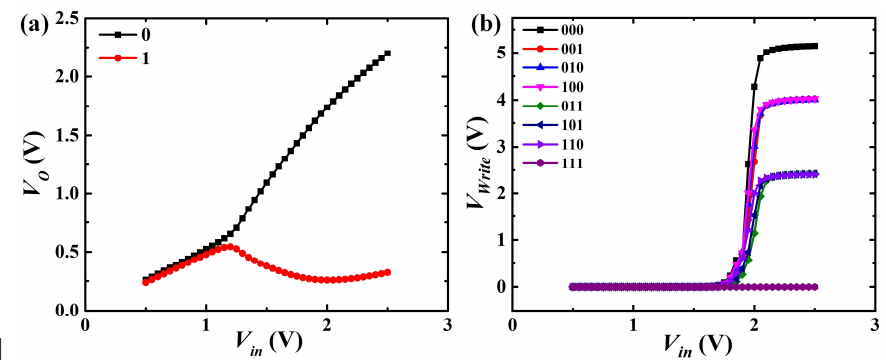

3 Fig. 2 Response of (a) the gate voltage of MOSFETs ( $\left.V_{O}\right)$ and (b) 4 write voltage of memory unit $\left(V_{\text {Write }}\right)$ to logic inputs 5

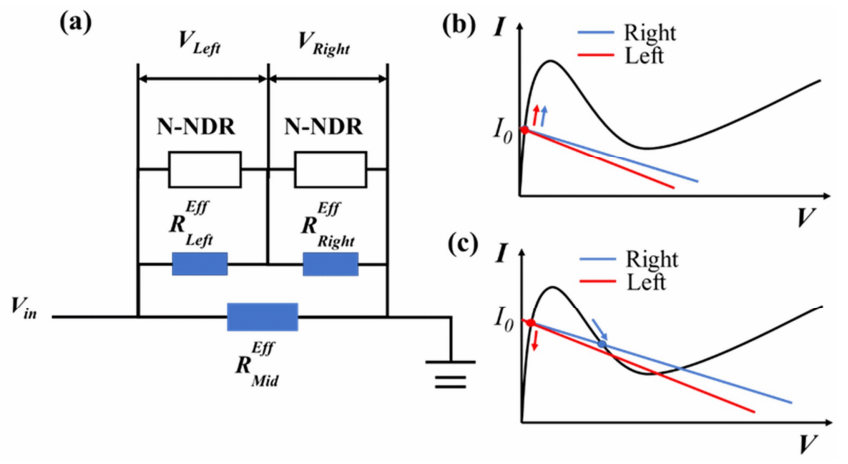

7

8 Fig. 3 (a) Modelling of three terminal magnetic bit by $\Delta$ - type 9 resistor network. (b)(c) Load line analysis of the mechanism of 10 NDR-induced enhancement of anomalous Hall voltage. 11

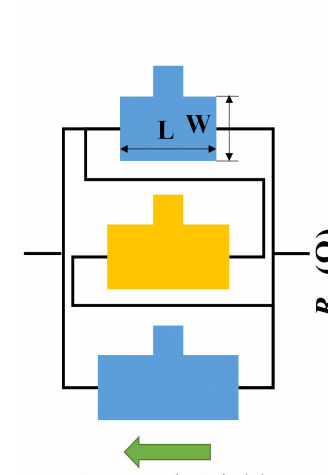

12 Magnetic Field

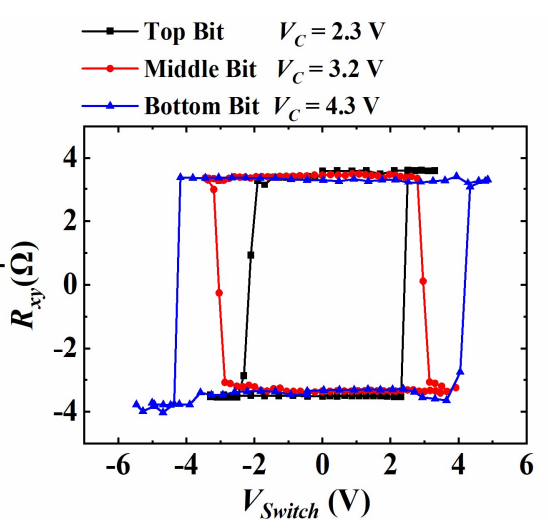

14 Fig. 4 Magnetization reversal of the magnetic bits in the memory 15 unit

16
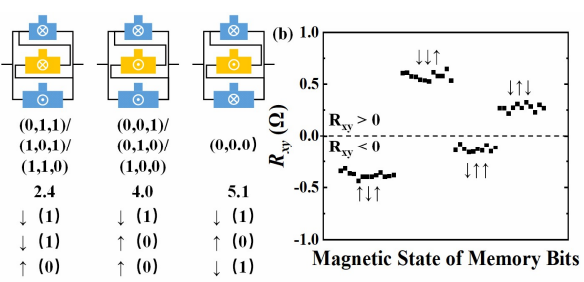

23

(a)

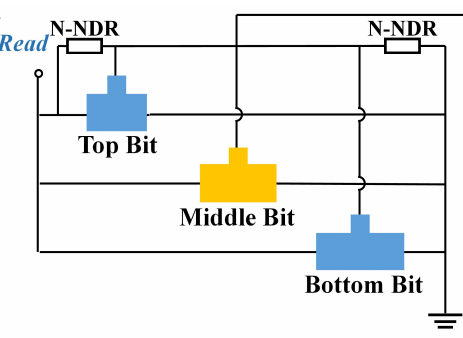

(c)
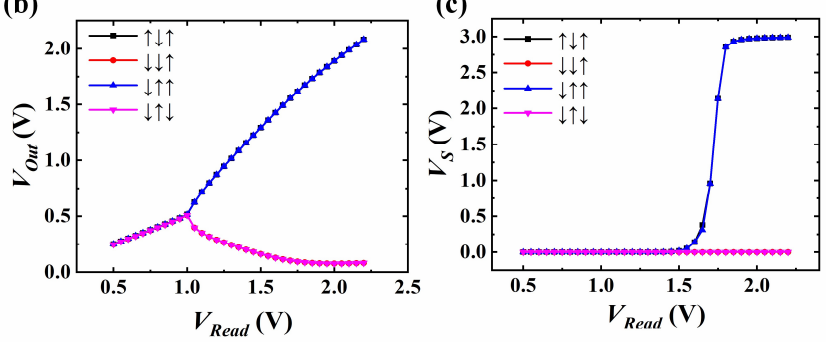

25 Fig. 6 (a) Read-out and rewriting circuit of "SUM" output. (b)(c) 26 NDR-enhanced anomalous Hall voltage $\left(V_{O u t}\right)$ and write voltage 27 of bit d $\left(V_{S}\right)$ corresponding to different magnetization of memory 28 unit. The NDR pair enhanced the AHE of the memory unit to 29 control the write voltage $V_{S}$ on bit d so that the "SUM" output was 30 rewritten into bit $\mathrm{d}$ for subsequent logic operations.

31

32 Table 1 Magnetization of Bit $\mathrm{d}$ under different logic inputs 33

\begin{tabular}{cccc}
\hline Inputs (a,b,c) & $V_{\text {Out }}(\mathbf{V})$ & $\boldsymbol{V}_{\boldsymbol{S}}(\mathbf{V})$ & $\begin{array}{c}\text { Magnetization } \\
\text { of Bit d }\end{array}$ \\
\hline$(1,1,1)$ & 2.08 & $2.99(1)$ & $1(\downarrow)$ \\
\hline$(1,1,0)$ & & \\
$(1,0,1)$ & 0.08 & $\sim 10^{-5}(0)$ & $0(\uparrow)$ \\
$(0,1,1)$ & & \\
\hline$(1,0,0)$ & & \\
$(0,1,0)$ & 2.08 & $2.99(1)$ & $1(\downarrow)$ \\
$(0,0,1)$ & & & \\
\hline$(0,0,0)$ & 0.08 & $\sim 10-5(0)$ & $0(\uparrow)$ \\
\hline
\end{tabular}

34

35

19 Fig. 5 (a) Four different magnetization states of memory unit with 20 different logic inputs. (b) Anomalous Hall effect of the memory 21 unit at the four magnetization states. 